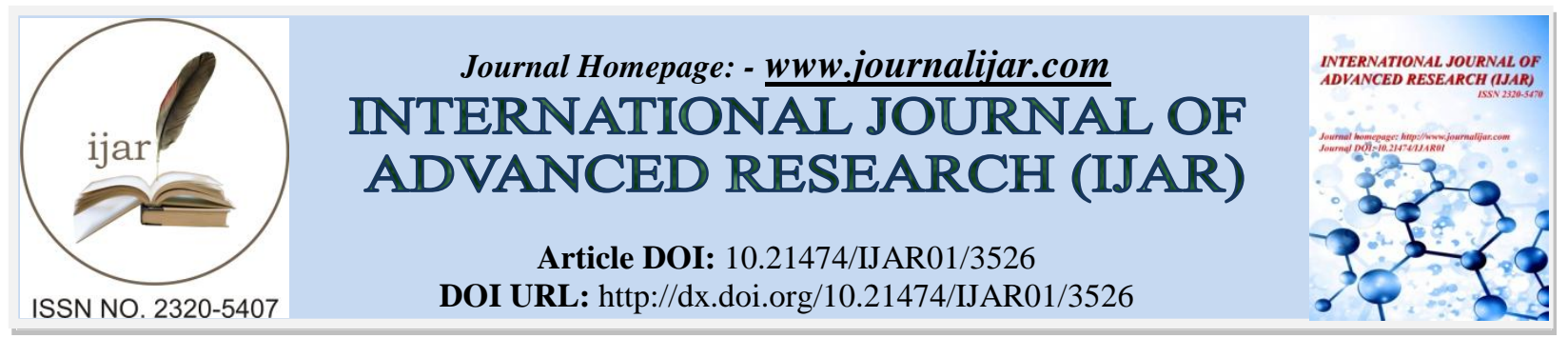

RESEARCH ARTICLE

\title{
FACILE SYNTHESIZE OF GRAPHENE OXIDE BY MODIFIED HUMMER'S METHOD AND DEGRADATION OF METHYLENE BLUE DYE UNDER VISIBLE LIGHT IRRADIATION.
}

\author{
B. Lavakusa ${ }^{1 *}$ B. Sathish Mohan ${ }^{1}$ P.Durga Prasad ${ }^{1}$, Neway Belachew ${ }^{1}$ and K.Basavaiah ${ }^{1 *}$ \\ ${ }^{1}$ Department of Inorganic \& Analytical Chemistry, Andhra University, Visakhapatnam, India -530003.
}

Manuscript History

Received: 19 January 2017

Final Accepted: 20 February 2017

Published: March 2017

Graphene oxide, Methylene blue, Degradation, Modified Hummer's method

\section{Manuscript Info}

Key words:-

\begin{abstract}
Graphene is a very huge and ultimate material for composite with metals, semiconductors and non metals in recent years, due to its has a unique atom-thick with two-dimensional (2D) structure, excellent physical properties like high conductivity and charge mobility, huge specific surface area, excellent mechanical, thermal and electrical properties. Thus, it has been regarded as an important component for functional materials, especially for developing a variety of catalysts and it has been considered widely as a prominent precursor and a starting material for the synthesis of this processable material. This work describes the synthesis of Graphene oxide (GO) by Modified Hummer's method and characterization of GO by Ultra violet visible spectroscopy (UV-Vis), UV-visible diffuse reflectance spectroscopy (UV-DRS), X-ray diffraction (XRD), Field emission scanning electron microscopy (FESEM) and Energy dispersive spectroscopy (EDX) analysis. The results obtained from the characterization techniques mentioned above is also explained in detailed and evaluation of catalytic application to environmental remedies, such as water purification of degradation of methylene blue under visible light irradiation.
\end{abstract}

Copy Right, IJAR, 2017,. All rights reserved.

\section{Introduction:-}

Graphene oxide (GO) represents an emerging field of interdisciplinary science that spans a variety of disciplines, including chemistry, physics, materials science, device fabrication, and nanotechnology [1-3] The current graphene boom started in 2004 when Geim, Novoselov et al. published the deposition and characterization of single sheets of graphite on solid supports. Their groundbreaking experiments on graphene were honored with the Nobel Prize in Physics in 2010 [4-7]. Exceptional electronic, optical and mechanical properties were discovered in quick succession as a consequence of the experience gained from other carbon allotropes [8-9]. In particular, the high charge carrier mobilities, the electrical and thermal conductivity, combined with transparency and mechanical strength make graphene highly attractive for future high-tech applications [30].

The current status of graphene oxide with respect to prototype applications has been extensively reviewed.[10] Many graphene-based devices outperform reference systems, for example in high-frequency transistors, foldable and stretchable electronic or photdetectors,[11] capacitors,[12] transparent electrodes, [13] sensors, [14] $\mathrm{H}_{2}$ generation,[15] pollution management,[16] energy applications,[17] biomedical applications and in composite materials [30].

Corresponding Author:- B. Lavakusa.

Address:- Department of Inorganic \& Analytical Chemistry, Andhra University, Visakhapatnam India - 
Graphene is a two-dimensional (2D) carbon allotrope which can be viewed as both a solid and a macromolecule with molecular weights of more than $10^{6}-10^{7} \mathrm{gmol}^{-1}$. In natural graphite, the graphene layers stick together through very pronounced $\pi-\pi$ stacking interactions [30]. This noncovalent interlayer binding contributes significantly to the high thermodynamic stability of graphite. As a consequence, the wet chemistry of graphene is always concerned with overcoming these interactions. For example, a targeted exfoliation of graphite or the stabilization of solventdispersed graphene sheets always competes with reaggregation. It should be pointed out that a solid sample of graphene can only be stabilized on a support, such as a surface. A nonsupported graphene powder can not be expected to exist, since at least a partial restacking to graphite will take place! Another possibility of stabilizing individualized graphene is to "mask" the surface through chemical functionalization[18,30]. This paper we reported catalytic activity of graphene oxide (GO) under visible light irradiation and comparison the catalytic activity with graphite flake.

\section{Properties of Graphene:- \\ Electronic properties:-}

The electronic properties of graphene have received the most attention. Suspended graphene shows extremely high low-temperature charge carrier mobility, with a value approaching $200000 \mathrm{~cm}^{2} \mathrm{~V}^{-1} \mathrm{~s}^{-1}$ [19], making it an outstanding conductor of electricity. This excellent conductivity is due to the remarkable property of graphene that its charge carriers behave as mass less relativistic particles or Dirac fermions, and electrons can travel distances in the order of micrometers without scattering under ambient conditions [21]. This unique behavior has led to other phenomena in graphene. UV-DRS spectra revealed the graphene oxide has a zero-band gap 2D semiconductor due to its like as a semi metal, graphene exhibits a strong ambipolar electric field effect and the concentration of charge carriers can be as high as $10^{13} \mathrm{~cm}^{-1}$, with room temperature mobilities of up to $15000 \mathrm{~cm}^{2} \mathrm{~V}^{-1} \mathrm{~s}^{-1}$ having been measured[22]. Moreover, an unusual half-integer quantum Hall effect (QHE) in graphene has been observed at room temperature[23,29].

\section{Thermal properties:-}

The thermal conductivity of suspended single-layer graphene at room temperature has been measured in the range $(4.84 \pm 0.44) \times 10^{3}$ to $(5.30 \pm 0.48) \times 10^{3} \mathrm{~W} \mathrm{~m}^{-1} \mathrm{~K}^{-1}$ [24]. The superior thermal conduction property of graphene is beneficial for making graphene as an excellent material for thermal management [29]. The thermal conductivity of large-area, monolayer graphene grown by chemical vapor deposition (CVD) on a Au-coated SiNx porous membrane can reach about $2500 \mathrm{~W} \mathrm{~m}^{-1} \mathrm{~K}^{-1}$ at $350 \mathrm{~K}[25,29]$.

\section{Mechanical Properties:-}

According to Changgu Lee, Graphene is the strongest material ever tested, with a Tensile strength of $130 \mathrm{GPa}$ and a Young's Modulus (defines stiffness) of $1 \mathrm{TPa}[22,32]$. Apart from this, Graphene is unbelievably light, weighing about only $0.77 \mathrm{mg} / \mathrm{m} 2$. According to a Nobel announcement which illustrates that $1 \mathrm{~m} 2$ of Graphene hammock would support a $4 \mathrm{~kg}$ cat, but would weigh only as much as one of the cat's whiskers [23,31].

\section{Optical Properties:-}

Graphene produces a highly opaque atomic monolayer in vaccum, as it has an ability to absorb approximately $2.3 \%$ of the white light. Adding another layer of Graphene increases the amount of white light absorbed by approx. the same value (2.3\%) [24]. Once optical intensity reaches a certain threshold saturable absorption takes place [33]. Due to Graphene's properties of wavelength-insensitive ultrafast saturable absorption, full band mode locking has been achieved [34].

\section{Experimental:- \\ Materials:-}

Graphite Flakes (Sigma Aldrich), Sodium nitrate $\left[\mathrm{NaNO}_{2}\right]$, Potassium permanganate $\left[\mathrm{KNO}_{3}\right]$, Hydrogen peroxide $30 \%\left[\mathrm{H}_{2} \mathrm{O}_{2}\right]$, Sulphuric acid $98 \%\left[\mathrm{H}_{2} \mathrm{SO}_{4}\right]$, Hydrochloric acid 35\% [ $\left.\mathrm{HCl}\right]$, Ortho phosphoric acid $\left[\mathrm{H}_{3} \mathrm{PO}_{4}\right], \mathrm{Bismuth}$ Nitrate $\left[\mathrm{Bi}\left(\mathrm{NO}_{3}\right)_{3} .5 \mathrm{H}_{2} \mathrm{O}\right]$, Nitric acid $\left[\mathrm{HNO}_{3} \quad 78 \%\right]$, Ammonium molybdate $\left[\left(\mathrm{NH}_{4}\right)_{6} \mathrm{Mo}_{7} \mathrm{O}_{24} .4 \mathrm{H}_{2} \mathrm{O}\right]$ and Ammonium hydroxide $\left[\mathrm{NH}_{4} \mathrm{OH}\right]$ and Methylene blue $\left[\mathrm{C}_{16} \mathrm{H}_{18} \mathrm{ClN}_{3} \mathrm{~S}\right]$. All chemicals received from Merck India except Graphite flake received from Sigma Aldrich USA and used without further purifier. 


\section{Synthesis of Graphene Oxide by modified Hummer's method:-}

Graphite flakes $(2 \mathrm{~g})$ and $\mathrm{NaNO}_{3}(2 \mathrm{~g})$ were mixed in $80 \mathrm{~mL}$ of $\mathrm{H}_{2} \mathrm{SO}_{4}(98 \%)$ and $10 \mathrm{ml}$ of $\mathrm{H}_{3} \mathrm{PO}_{4}$ in a $1000 \mathrm{ml}$ round bottom flask kept under at ice bath $\left(0-5^{\circ} \mathrm{C}\right)$ with continuous stirring. The mixture was stirred for 4 hrs at 0 $5^{\circ} \mathrm{C}$ temperature and potassium permanganate $(12 \mathrm{~g})$ was added to the suspension very slowly. The rate of addition was carefully controlled to keep the reaction temperature lower than $15^{\circ} \mathrm{C}$. The mixture is diluted with very slow addition of $200 \mathrm{ml}$ double distilled water, the ice bath was then removed and kept under stirring for $2 \mathrm{hrs}$ at room temperature. The above mixture is kept in a reflux system at $90^{\circ} \mathrm{C}$ for $1 \mathrm{~h}$. After $1 \mathrm{~h}$, change the temperature to $30^{\circ} \mathrm{C}$ which gives brown colored solution. Again after $10 \mathrm{~min}$, change it to $25^{\circ} \mathrm{C}$, and maintain the temperature for $2 \mathrm{hrs}$. The solution is finally treated with $40 \mathrm{ml} \mathrm{H}_{2} \mathrm{O}_{2}$ by which color changes to bright yellow, $200 \mathrm{ml}$ of water is taken in two separate beakers and equal amount of solution prepared is added and stirred for $1 \mathrm{hr}$. It is then kept without stirring for 3-4 hrs, where the particles settles at the bottom and remaining water is poured to filter. The resulting mixture is washed repeatedly by centrifugation with $5 \% \mathrm{HCl}$ and then with distilled water several times until it forms gel like substance (pH- neutral). After centrifugation the gel like substance is vacuum dried [35].

\section{Photocatalytic test:-}

Organic pollutant such as methylene blue were chosen for photocatalytically activities evaluate of the as synthesized GO powder and Graphite flake (Sigma Aldrich USA). A $400 \mathrm{~W} \mathrm{HgX}$ lamp was used as the light source to provide the simulated solar light. The experiments performed at an ambient temperature. The photocatalysts (GO or Graphite flake) $0.05 \mathrm{~g}$ were added into $50 \mathrm{~mL}$ of organic pollutant $5 \mathrm{ppm}$ solution, and suspension was magnetically stirred for $30 \mathrm{~min}$ in the dark to reach an adsorption-desorption equilibrium with the photocatalysts. At a every 30 min time intervals, a $3 \mathrm{~mL}$ solution collected and then analyzed on UV-Vis spectrophotometer during the photo degradation process and same experiment perform as blank (without GO or Graphite flake). The concentration of methylene blue were determined by monitoring the variations in the main absorption centered at $664 \mathrm{~nm}$.

\section{Characterization:-}

The crystalline structure of the Graphene Oxide (GO) was investigated by powder X-ray diffraction (XRD, PanAlytical, X-Pert pro, Netherland ) with $\mathrm{Cu} \mathrm{K} \alpha(\lambda-1.54218$ A) radiation. The size and morphology of the product were obtained with a Scanning electron microscope (FESEM-CARL Zeiis Germany, Model Ultra 55 FESEM, Gemini column, 1nm Resolution, detector is INLENSE ), Energy dispersive spectroscopy (Oxford company, model $20 \mathrm{~nm} \mathrm{X} \mathrm{Ma)} \mathrm{UV-Vis} \mathrm{\&} \mathrm{UV-Vis} \mathrm{diffuse} \mathrm{reflectance} \mathrm{spectra} \mathrm{of} \mathrm{the} \mathrm{sample} \mathrm{were} \mathrm{obtained} \mathrm{in} \mathrm{the}$ range $300-800 \mathrm{~nm}$ using a UV-vis spectrophotometer (UV2550, Shimadzu, Japan) barium sulphate $\left(\mathrm{BaSO}_{4}\right)$ was used as a reflectance standard.

\section{Results and Discussion:-}

Figure.1. shows the UV-Visible spectra of Graphene Oxide, the peak at $215 \mathrm{~nm}$ is $\pi-\pi^{*}$ transition due to $\mathrm{C}=\mathrm{C}$ bonding is an aromatic ring, where as the broad shoulder peak at $291 \mathrm{~nm}$ is $\mathrm{n}-\pi^{*}$ transition due to C-O bonding. The absorption over $291 \mathrm{~nm}$ is expected to be caused by the conjugated fused ring plane; this indicates a $\mathrm{sp}^{2}-\pi$ conjugated network.

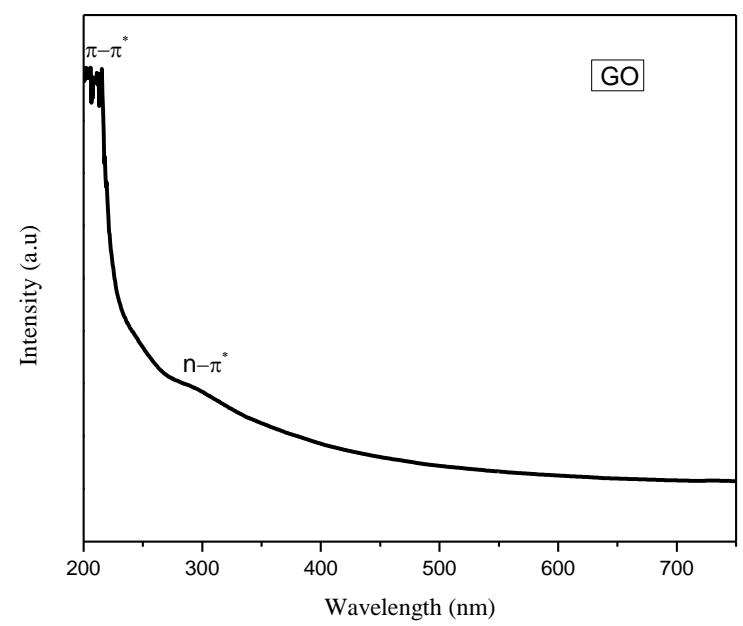

Fig.1:- UV-Visible spectra of Graphene Oxide 


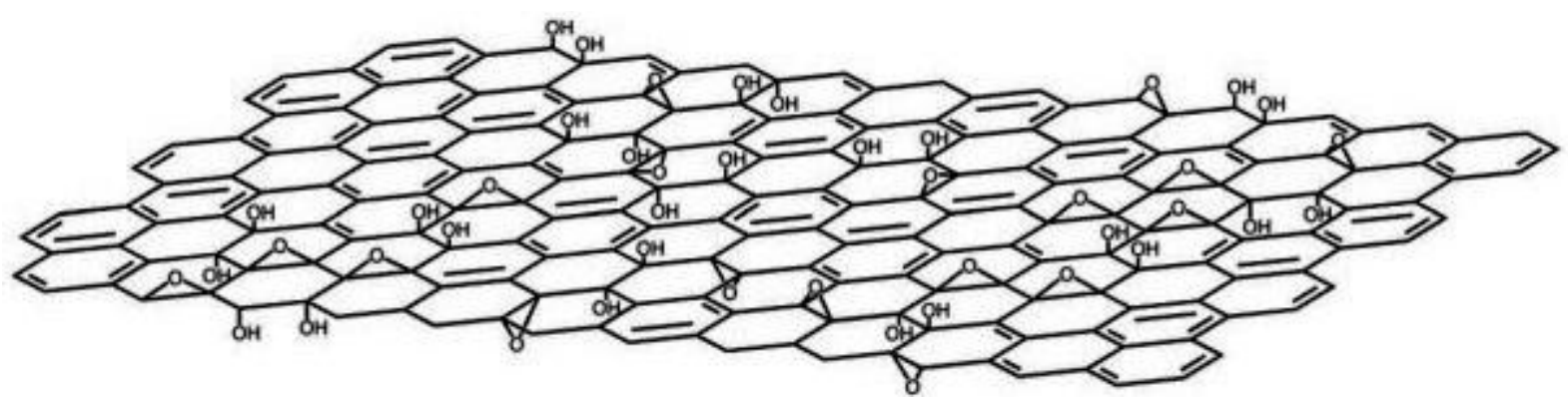

Fig 2:- Possible structure of Graphene Oxide

The XRD pattern obtained for as synthesized GO nanoparticles by Hummer's method is shown in Figure 3b. It shows the diffraction peak at $2 \theta=10^{\circ}$, which is mainly due to the oxidation of graphite. The diffraction peak of pure graphite is found around $26^{0}$, corresponding to the highly organized layer structure with an interlayer distance of $0.34 \mathrm{~nm}$ along the (002) orientation is shown as inset in Figure 3a. The XRD pattern for synthesized GO by Modified Hummer's method is shown in Figure 3b. The disappearance of the peak at $26^{\circ}$ and appearance of the peak at $10^{\circ}$, shows that the product is completely oxidized after the chemical oxidation and exfoliation, indicating an increase in $\mathrm{d}$-spacing from $0.34 \mathrm{~nm}$ to $0.82 \mathrm{~nm}$.

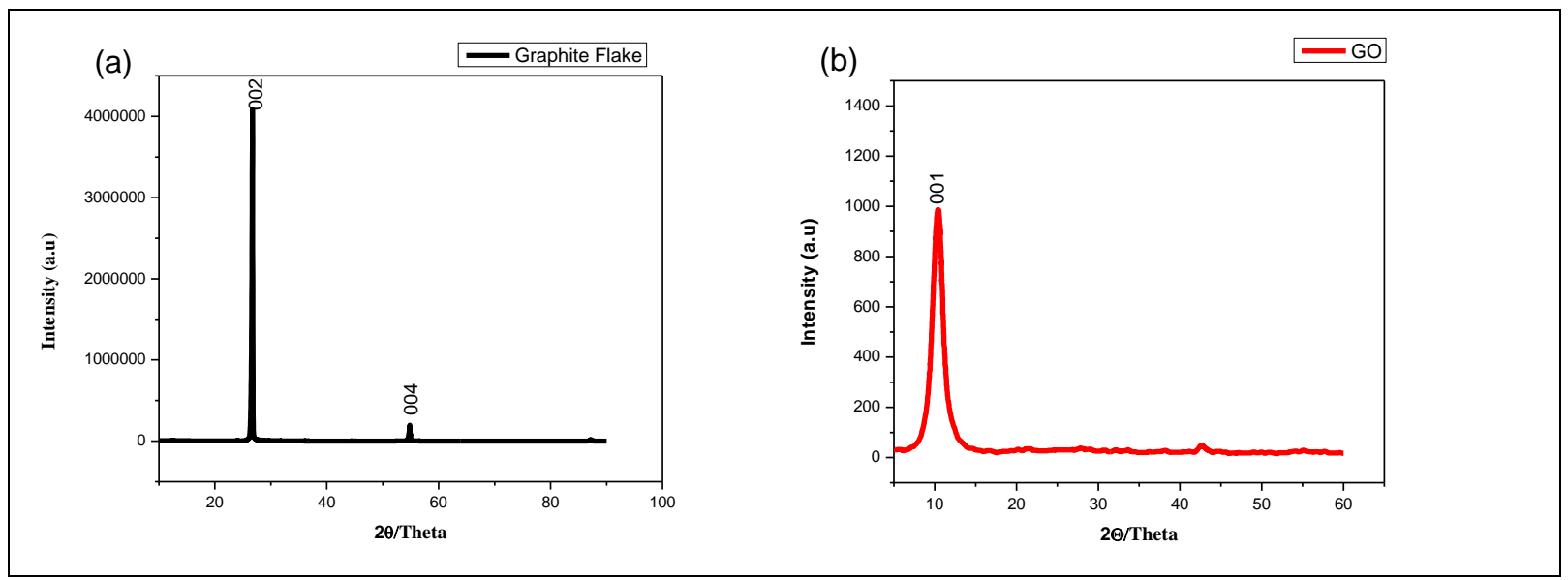

Fig.3:- XRD pattern of (a) Graphite flake, (b) Graphene Oxide.

Figure .4. Shows the FESEM images of Graphene Oxide. It shows single flakes of Gaphene Oxide may be observed. Graphene Oxide flakes have relatively large surface and well defined and interlinked three-dimensional Graphene Oxide sheets, forming a porous network that resembles a loose sponge like structure with $49.38 \mathrm{~nm}$ range in size. Fig.5.shows the EDX spectra of Graphene Oxide, which revealed to purity of C,H,O elemental analysis and some impure of the elemental with Potassium (K) and Sulphur (S) also idetified due $\mathrm{KMnO}_{4}$ and $\mathrm{H}_{2} \mathrm{SO}_{4}$ are precursor for the oxidation of GO.. 


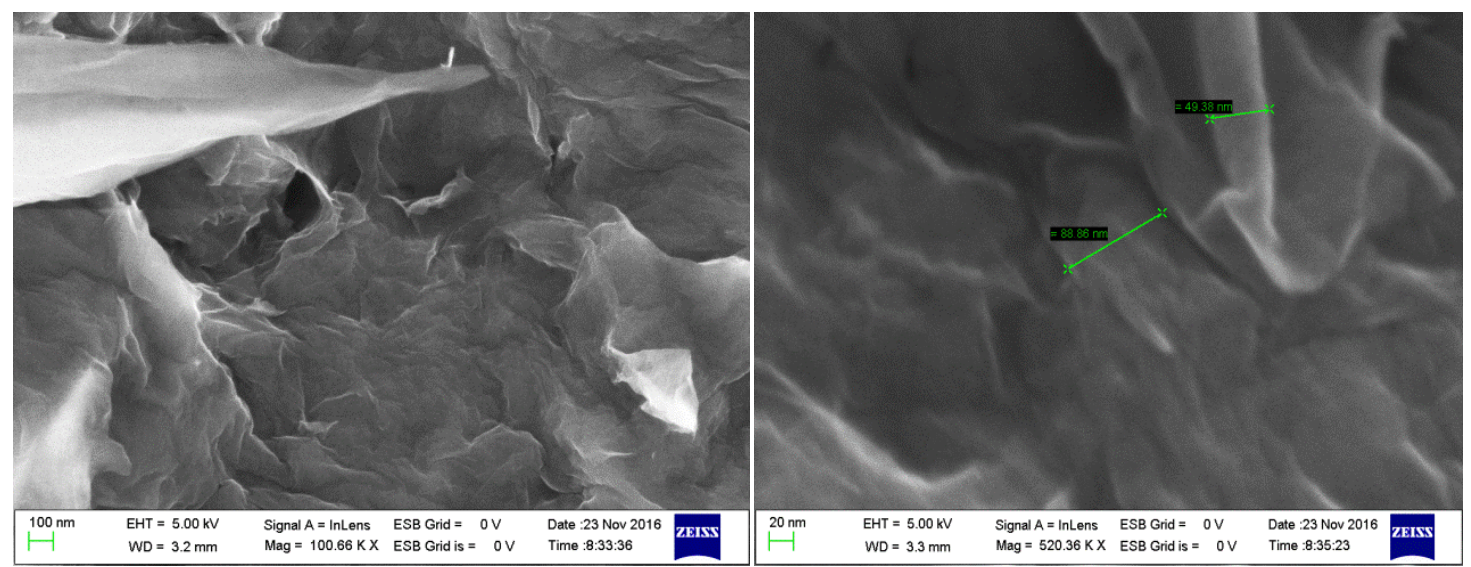

Fig 4:- FESEM images of Graphene Oxide

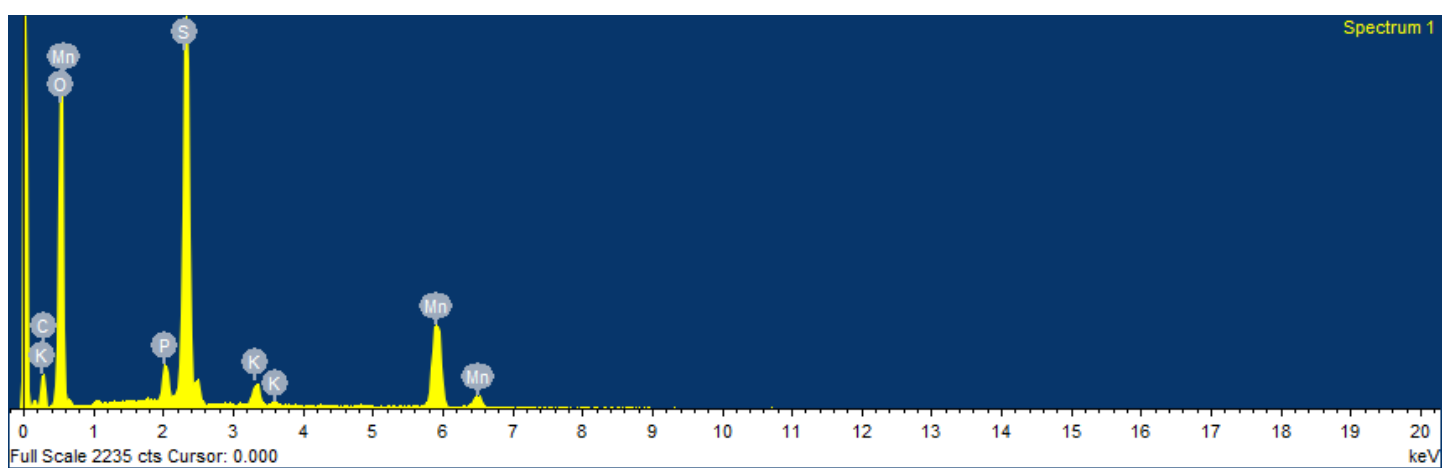

Fig 5:- EDX spectra of Graphene Oxide.

Fig.6. shows Ultra Violet Diffuse-Reflectance Spectroscopy (UV-DRS) is an important method for charactering the electronic state, optical state and calculates the band gap of semiconductor materials. The DRS spectra of as synthesized of Graphene Oxide and the band gap calculated and the band gap is zero, due to its revealed Graphene Oxide as a non semiconductor, its like metal so Graphene Oxide as a semi metal.

Band gap energy $\left(E_{g}\right)=h * c / \lambda$

Where $E_{g}, h, c, \lambda$ are band gap energy, plank constant, speed of light, cut of wavelength respectively. 


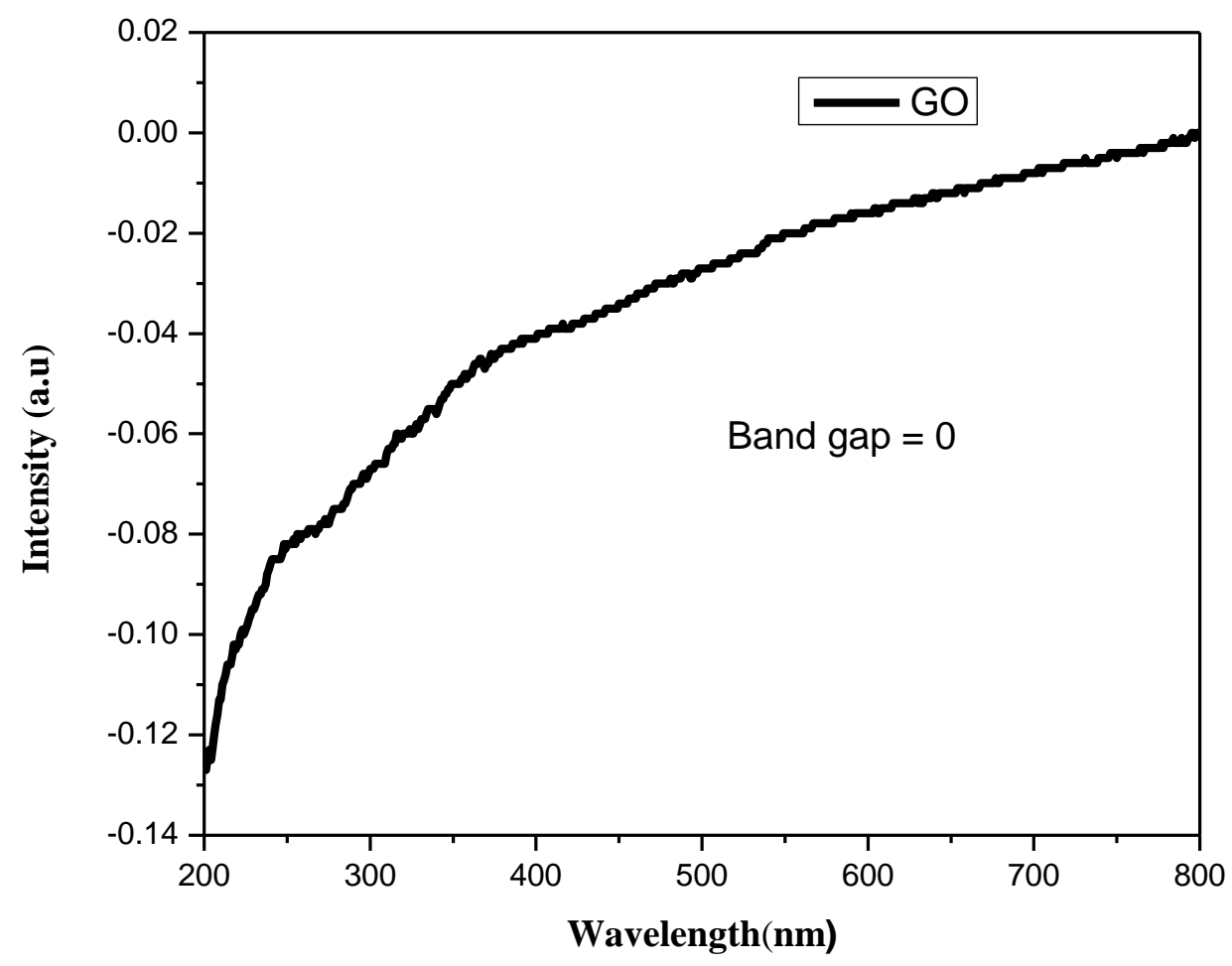

Photocatalytic Effect:-

Fig 6:- UV-DRS spectrum of Graphene Oxide

Generally photocatalytic degradation of dyes by a photocatalyst is critically depends on particle size, crystallinity, morphology, band gap energy, charge recombination and efficient charge separation. Graphene Oxide as a good efficiency for charge separation. Fig.7. shows the photocatalytic activity under visible light irradiation over degradation of Methylene blue were chosen as representative model pollutant. The establishment of adsorption desorption equilibrium was obtained under continuous stirring $30 \mathrm{~min}$ before the degradation reaction was carried out, evaluated degradation under visible light irradiation measured to UV-Visible spectrometer to the cut off filter $664 \mathrm{~nm}$. Fig. 7a. shows $95.69 \%$ degradation of MB with GO, Fig.7b shows $58.02 \%$ degradation of MB with Graphite Flake and Fig.7c shows the 7.6\% degradation of MB in absence of catalyst within 120 min. Fig 7d shows a plot between time verses \% of degradation of MB under GO, Graphite flake and in absence of catalyst. Among them GO responds more towards the degradation of MB under visible light irradiation and the GO curve indicate with increase the time degradation also increased, the increases of dye degradation below 30 minutes is rapidly after 30 minutes the degradation of dye is slow due to Graphene Oxide has large surface area so the degradation of starting 30 minutes is rapidly. So the effect of dye degradation not only depend on photo light (visible or ultra violet) also depend on surface area of catalyst.

$\%$ of degradation $=\left(\mathrm{A}_{0}-\mathrm{A}_{\mathrm{t}}\right) / \mathrm{A}_{0} * 100$

Where $A_{0}$ and $A_{t}$ are respectively initial absorbance. 

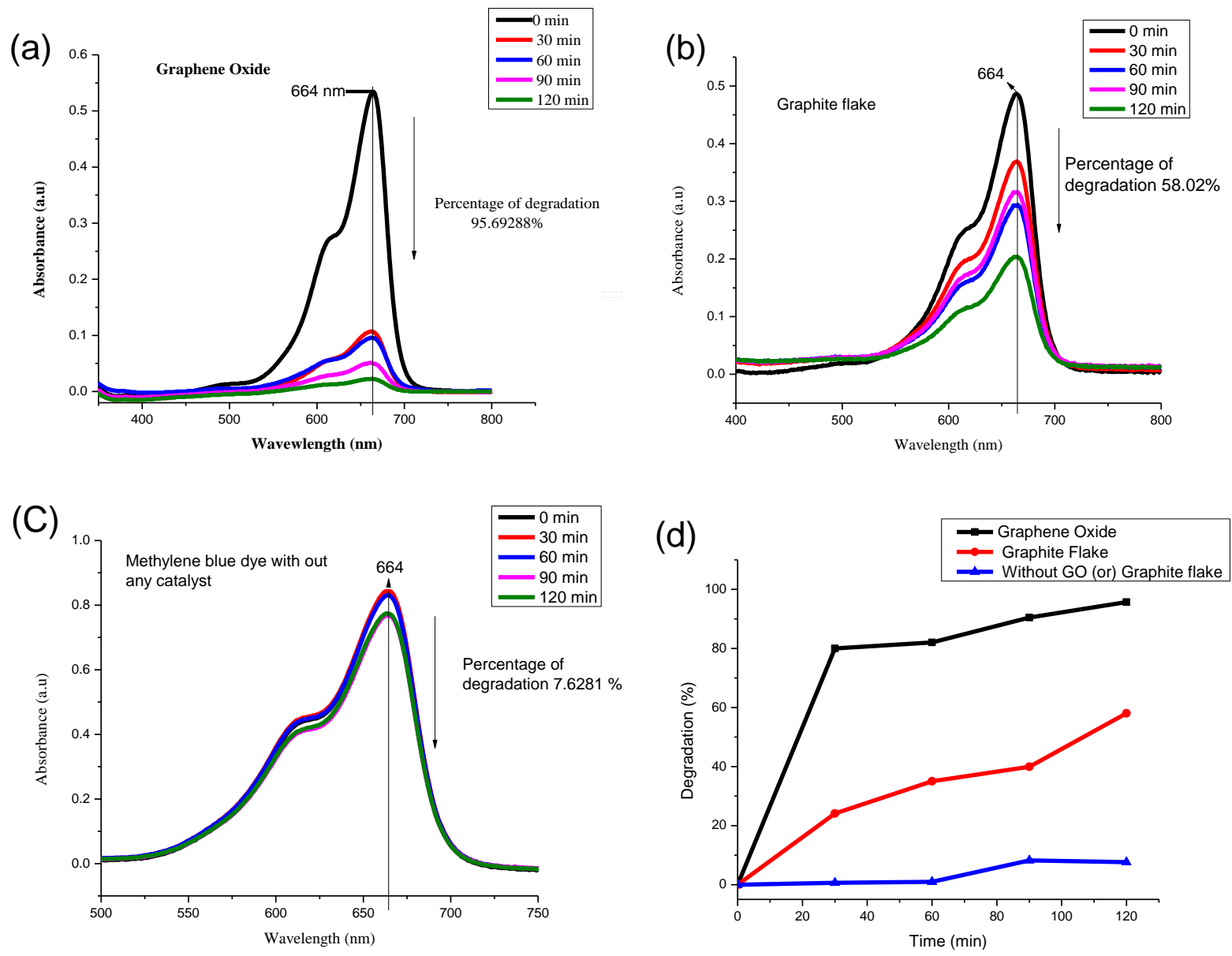

Fig.7:- Degradation of methylene blue dye under Visible light irradiation (a) MB dye with GO (b) MB dye with Graphite flake (c) MB dye without catalyst (d) Photocatalytic comparison between GO, Graphite flake and without catalyst under visible light irradiation as a function of time ' $t$ ' verses $\%$ of degradation.

\section{Conclusion:-}

The synthesis of Graphene Oxide successfully synthesized by modified hummer's method. The synthesized of Graphene Oxide confirmed by XRD for the graphite flake disappearance of the peak at $26^{\circ}$ and appearance of the peak at $10^{\circ}$, shows that the product is completely oxidized after the chemical oxidation to formation of Graphene Oxide and presence of $\mathrm{C}-\mathrm{O}\left(\mathrm{n}-\pi^{*}\right.$ transition $), \mathrm{C}=\mathrm{C}\left(\pi-\pi^{*}\right.$ transition)bonds analysis by UV-Visible spectroscopy, FE-SEM image revealed sponge porous with large surface area and photocatalytic experiment gave good information for degradation of methylene blue dye with Graphene Oxide as a catalyst i.e. the effect of dye degradation not only depend on photo light (visible or ultra violet) also depend on surface area also due to this reason Graphene Oxide as only one best material for composite/hybridized to semiconductors and metals.

\section{Acknowledgment:-}

Author gratefully acknowledge to the UGC, New Delhi for financial support of this work and DST-FIST instrument laboratory, Department of Inorganic \& Analytical Chemistry, Andhra University for providing instrumentation facility. 


\section{Reference:-}

1. K. S. Novoselov, A. K. Geim, S. V. Morozov, D. Jiang, Y. Zhang, S. V. Bubonos, I. V. Grigorieva, A. A. Firsov, Science. 2004, 306, 666

2. K. S. Novoselov, A. K. Geim, S. V. Morozov, D. Jiang, M. I. Katsnelson, I. V. Grigorieva, S. V. Dubonos, A. A. Firsov, Nature. 2005, 438, 197

3. K. S. Novoselov, D. Jiang, F. Schedin, T. J. Booth, V. V. Khotkevich, S. V. Morozov, A. K. Geim, Proc. Natl. Acad. Sci. USA. 2005, 102, 10451

4. M. I. Katsnelson, K. S. Novoselov, A. K. Geim, Nat. Phys. 2006, 2, 620

5. K. S. Novoselov, E. McCann, S. V. Morozov, V. I. Fal'ko, M. I. Katsnelson, U. Zeitler, D. Jiang, F. Schedin, A. K. Geim, Nat. Phys. 2006, 2, 177

6. A. K. Geim, Angew. Chem. 2011, 123, 7100; Angew. Chem. Int. Ed. 2011, 50, 6966

7. K. S. Novoselov, Angew. Chem. 2011, 123, 7123; Angew. Chem. Int. Ed. 2011, 50, 6986

8. A. H. C. Neto, N. M. R. Peres, K. S. Novoselov, A. K. Geim, Rev. Mod. Phys. 2009, 81, 109

9. C. N. Lau, W. Bao, J. Velasco, Mater. Today. 2012, 15, 238

10. M. J. Allen, V. C. Tung, R. B. Kaner, Chem. Rev. 2010, 110, 132

11. H. Chen, X. Guo, Small 2013, 9, 1144

12. Y. Huang, J. Liang, Y. Chen, Small 2012, 8, 1805

13. D. Chen, H. Feng, J. Li, Chem. Rev. 2012, 112, 6027

14. G. Xie, K. Zhang, B. Guo, Q. Liu, L. Fang, J. R. Gong, Adv. Mater. 2013, 25, 3820

15. K. L, G. Zhao, X. Wang, Chin. Sci. Bull. 2012, 57, 1223

16. B. Luo, S. Liu, L. Zhi, Small. 2012, 8, 630

17. C. Chung, Y. K. Kim, D. Shin, S. R. Ryoo, B. H. Hong, D. H. Min, Acc. Chem. Res. 2013, 46, 2211

18. J. I. Paredes, S. Villar-Rodil, A. Martnez-Alonso, J. M. D. Tascn, Langmuir. 2008, 24, 10560

19. K. I. Bolotin, K. J. Sikes, Z. Jiang, M. Klima, G. Fudenberg, J. Hone, P. Kim and H. L. Stormer, Solid State Commun. 2008, 146, 351-355

20. K. S. Novoselov, A. K. Geim, S. V. Morozov, D. Jiang, Y. Zhang, S. V. Dubonos, I. V. Grigorieva and A. A. Firsov, Science. 2004, 306, 666-669

21. Y. Zhu, S. Murali, W. Cai, X. Li, J. W. Suk, J. R. Potts and R. S. Ruoff, Adv. Mater. 2010, 22, 3906-3924

22. K. S. Novoselov, A. K. Geim, S. V. Morozov, D. Jiang, Y. Zhang, S. V. Dubonos, I. V. Grigorieva and A. A. Firsov, Science. 2004, 306, 666-669

23. K. S. Novoselov, Z. Jiang, Y. Zhang, S. V. Morozov, H. L. Stormer, U. Zeitler, J. C. Maan, G. S. Boebinger, P. Kim and A. K. Geim, Science. 2007, 315, 1379

24. J. H. Seol, I. Jo, A. L. Moore, L. Lindsay, Z. H. Aitken, M. T. Pettes, X. Li, Z. Yao, R. Huang, D. Broido, N. Mingo, R. S. Ruoff and L. Shi, Science. 2010, 328, 213-216

25. W. Cai, A. L. Moore, Y. Zhu, X. Li, S. Chen, L. Shi andR. S. Ruoff, Nano Lett. 2010, 10, 1645-1651

26. Lee, Changgu, Science 2008, 321 (385): 385- 388

27. "2010 Nobel Physics Laureates", www.nobelprize.org

28. Nair, R. R.; Blake, P.; Grigorenko, A. N.; Novoselov, K. S.; Booth, T. J.; Stauber, T.; Peres, N. M. R.; Geim, A. K. Science 2008, 320 (5881): 1308-1308

29. J. Mater. Chem. A, 2014, 2, 4491

30. Angew. Chem. Int. Ed. 2014, 53, $7720-7738$

31. 31. Mattei, Tobias A. MD*; Rehman, Azeem A. BS ${ }^{\star}$ Neurosurgery, 2014 - Volume 74 - Issue 5 - p $499-516$

32. en.wikipedia.org

33. Santosh K. Tiwari,Vijay Kumar, Andrzej Huczko, R. Oraon, A. De Adhikari, G. C. Nayak. Critical Reviews in Solid State and Materials Sciences 201641 (4), 257-317

34. www.graphenea.com

35. Paulchamy et al., J Nanomed Nanotechnol 2015, 6:1 